\title{
Morphological Characterization of Mango (Mangifera indica L.) Germplasm Using DUS Testing
}

\author{
Kanchan Bhamini $^{1 *}$, Anjani Kumar ${ }^{2}$, U.S. Jaiswal ${ }^{1}$, Md. Feza Ahmad ${ }^{1}$ and Ruby Rani ${ }^{1}$ \\ ${ }^{1}$ Department of Horticulture (Fruit and Fruit Technology), Bihar Agricultural University \\ Sabour Bhagalpur, Bihar-813 210, India \\ ${ }^{2}$ Department of Plant Breeding and Genetics, Bihar Agricultural University Sabour \\ Bhagalpur, Bihar-813 210, India
}

*Corresponding author

\section{A B S T R A C T}

\begin{tabular}{|l|}
\hline Ke y w o r d s \\
$\begin{array}{l}\text { Mango varieties, } \\
\text { morphological } \\
\text { parameters, flower, } \\
\text { descriptors and } \\
\text { qualitative characters }\end{array}$ \\
\hline Article Info \\
\hline $\begin{array}{l}\text { Accepted: } \\
\text { 20 April } 2018 \\
\text { Available Online: } \\
\text { 10 May } 2018\end{array}$ \\
\hline
\end{tabular}

\section{Introduction}

The world needs to increase crop productivity for the development of the valuable varieties to changing environmental and biological challenges that meets to evolve the needs of local communities. To meet these needs and challenges, farmers and scientists not only must have access to a wide range of plant genetic resources but also must have access to the essential information about those plant genetic resources that will allow effective used
Studies were carried out on mango varieties developed at Horticulture Garden, BAU Sabour to develop and document descriptors, during 2016-2017. The experiment was laid out in row column design with three replications having 24 varieties as treatments. Observations were recorded on various morphological parameters such as qualitative characters. From the observations made it was found that the mango varieties expressed the morphological variations from genotype to genotype. These morphological variations have the ability to distinguish between the mango varieties with each other. Descriptors were developed for 24 mango genotypes after observations. Among these genotypes, 5 morphological descriptors were developed and documented, which consist of 27 qualitative characters. Among the characters, five characters were developed for plant height, eight characters for leaf, five characters for inflorescence, five characters for fruit and four characters for fruit stones. The developed and documented descriptors will facilitate in plant variety registration, identification of genotypes and also in tree improvement programmes. 
information about the origin, characterization, and performance of germplasm is essential for effective conservation and use (Biodiversity, 2007). Success of commercial cultivation of Mango is much dependent on use of high yielding genotypes "instead" of low yielding local genotypes. Therefore, genetic improvement of this crop is quite indispensable to develop high-yielding genotypes. Selection/ development of high yielding genotypes depend on the genetic variability present in the gene pool.

Mango (Mangifera indica L.) is the most nutritive and delicious fruit crop belonging to the Anacardiaceae family and originated in Indo-Burma region. It is a commercial fruit crop of tropical and subtropical climatic condition which occupies an important socioeconomic position in India and south-east Asian regions. It is a tetraploid species $(4 \mathrm{x})$ species having chromosome number 40 . The cultivation of mango is believed to have started four to six thousand years back rendering it as old as the Indian civilization itself (Mukherjee, 1953; Kostermans and Bompard, 1993). It is recognized as the pride fruit of India, being the richest source of vitamin A (4800 I.U.) and good source of vitamin $\mathrm{C}$, minerals and other nutrients. In India, mango is currently grown in an area of 2.52 million hectares and it is the largest producer of mango (18.43 million tons) in the world but its average productivity (7.3 metric tons per hectare) (NHB, 2015). In the past mango genotypes have been characterized by usually morphological traits which are used for diversity assessments (Singh, 1969) and isozymes (Degani et al., 1992), though easy but are not reliable. The inferences derived on the basis of morphological assessment can be misleading as they are prone to change in response to environmental fluctuations (Sankar et al., 2011). The crop improvement program mainly depends on the choice of superior parents for hybridization and the knowledge of combining ability and magnitude of gene action involved in the expression of important traits.

Characterization of germplasm is essential to maximize its utility (Rubenstein et al., 2006) and foster efficient ex-situ conservation (Hammer et al., 2003 and De Vicente et al., 2006). The evaluation of morphological and agronomic characters can provide relevant information on yield and quality traits, as well as other information of great interest to breeders, such as descriptions of available variation and estimates of trait heritability (Anumalla et al., 2015). Phenotypic characters are mainly influenced by environments and plant developmental stages. In addition, species with similar morphological characters cannot be easily distinguished. DUS test apply to all varieties of mango (Mangifera indica L.) for testing the variety and when and where it is to be delivered for registration under the Protection of Plant Variety and Farmers' Rights (PPV \& FR) Act, 2001. It is based on the quantity and quality of the plant materials which is required for testing the varieties. In this study, an effort has been made to generate and identify DUS test to investigate structure of mango germplasm of India with special emphasis on genotype's collection. For an optimal characterization of genetic resources and for valid data comparisons among trials, it is necessary to use standard descriptors, like those developed by Biodiversity International for other fruit crops like Citrus and Apple etc., (Biodiversity, 2007) or by NBPGR in India. Hence, documentation of the variation in the morphological traits is necessary to effectively tap the available diversity in the crop improvement programmes. Against this background, an attempt has been made to characterize and categorize, the morphological diversity in the germplasm collected and conserved from all over India, in the form of minimal descriptors of Mango. Comprehensive information regarding Mango 
variability as required by the breeder for evaluation and characterization of various traits, to enhance its productivity and commercial value is lacking. The best efforts were put in describing the plant taking into consideration all the important agro morphological traits derived from a large germplasm collection maintained at an experimental plot of Horticulture Garden, Bihar Agriculture College, Sabour, Bhagalpur, India. This is a first and initial attempt to developing and documents the diversity pictorially in Mango. There is further scope for addition on the traits, where variation within genotypes has observed. However, it is hoped that the descriptor will serve as a reference to characterize the available diversity to a large extent.

\section{Materials and Methods}

The present investigation was carried out during 2016-2017 at Experimental Field of Horticulture Garden of Bihar Agricultural College, Sabour a campus of Bihar Agricultural University, Sabour Bhagalpur. Sabour is located about $10 \mathrm{~km}$ east of Bhagalpur city in Bihar state of India. It is situated at longitude $87^{\circ} 2$ ' 72 ' east and latitude $25^{\circ} 15^{\prime} 40$ " north at an altitude of 46 meters above mean sea level in the heart of the vast the vast Indo-Gangatic plain of North India, South of river Ganga. The experiment consisted of 24 treatments i.e., Bombai, Rani Pasand, Husn-e-ara, Dalma, Sundar Prasad, Baramasia, Taimuria, Peri Poona, Alphanso Poona, Mahmood Bahar, Alfazli, Sabri, Jawahar, Prabhashankar, Menka, Dada Miyan, Kesington, Kurrkkane, Mylepelian, Kesar, Mulgoa, Vanraj, Neeludin and Sital Pasand, respectively were selected for investigation. The site enjoys an average annual rainfall 34.9 $\mathrm{mm}$. The mean maximum and minimum temperature are $31.08{ }^{0} \mathrm{C}$ and $17.34{ }^{0} \mathrm{C}$, respectively. The soils were classified as slightly alkaline with $7.65 \mathrm{pH}$. Data were collected on morphological (qualitative) characters using character descriptors. Type of assessment of characteristics was as follows (UPOV, 1989).

VG: Visual assessment by a single observation of a group of plants or parts of plants.

VS: Visual assessment by observation of individual plants or parts of plants.

\section{Descriptors}

The requirement of Distinctiveness, uniformity and stability were assessed on the basis of descriptors. The descriptors are a feature of whole plant or part of plant. Such descriptors may be morphological, biochemical, molecular or any other nature. Mainly three types of descriptors are recommended. In the present study a total of 27 morphological characters, which correspond to the different plant characteristics and plant parts, including leaves, flowers and inflorescences, fruits and seeds, and overall architecture have been established.

\section{Qualitative descriptors}

Qualitative descriptors are those that are expressed in discontinuous states. These states are self-explanatory and independently meaningful. All states are necessary to describe the full range of the characteristics and every form of expression can be described by a single state. The qualitative descriptors studied were as follows:

\section{Plant}

Plant type (recorded as seedling and grafted); Branching pattern (the position of origination of branches from the stem was observed and recorded as Basal, Intermediate and Top); Plant growth habit (recorded as Erect, 
Spreading, Drooping, Very vigorous, Semivigorous and Dwarf based on the observations made); Crown shape (the shape of crown was found different and recorded as oblong, broadly pyramidal, semi-circular, spherical and others) and Foliage density (density of foliage was observed and recorded as Sparse, Intermediate and Dense).

\section{Leaf}

Young leaf colour (By visual observations, colour of the leaves were decided and tabulated as Light green, Light green with brownish tinge, Light brick red, Reddish brown and Deep coppery tan) and Mature leaf colour (Colour of mature leaf was observed and recorded as different colours such as Pale green, Green and Dark green); Leaf blade shape (Different leaf blade shapes were tabulated as Elliptic, Oblong, Ovate, Obovate, Lanceolate and Oblanceolate; Leaf apex shape (Different apex shape was also observed and recorded as Obtuse, Acute and Acuminate); Leaf base shape (recorded as Acute, Obtuse and Round); Leaf margin (Leaf margin were recorded as Entire and Wavy) and Angle of secondary vein to mid rib (Leaf venation angles were tabulated as Narrow: $<45^{\circ}$, Medium: $45-60^{\circ}$ and Wide: $>60^{\circ}$ ). Texture: The texture of the leaves were observed and also tabulated as Coriaceous, Chartaceous and Membranous.

\section{Inflorescence}

Inflorescence density (Flower density was observed and recorded as Sparse, Medium and Dense); Inflorescence colour (Flower colour was observed and tabulated as Whitish, Yellowish green, Yellow, Light green, Green with red patches, Light orange, Pink, Dark Pink, Purple, Light red, Red, Dark red and Crimson); Inflorescence position (position of inflorescence was observed and tabulated as Axillary and Terminal); Inflorescence axis growth (Different Inflorescence axis growth observed and tabulated as Semi-erect, Horizontal and Drooping) and Inflorescence shape (recorded as Conical, Pyramidal and Broadly pyramidal).

\section{Fruit}

Fruit colour (colour of the fruits were observed and tabulated as Green, Greenish yellow, Yellow, Green with red blush, Yellow with red blush and Greenish with purple patches); Fruit shape (fruit shape was observed and tabulated as Round, Oval and Oblong); Fruit size (size of the fruits were observed and recorded as Small, Medium, Large and Long), Fruit beak type (tabulated as Perceptible, Pointed, Prominent and Mammiform) and Fruit sinus type (recorded as Absent, Shallow and Deep).

\section{Fruit stone}

Veins on stone (Different stone veins were observed and tabulated as Level with surface, Depressed and Elevated); Pattern of stone venation (Stone venation pattern was recorded as Parallel and Forked); Quantity of fibre on stone (recorded as Low, Intermediate and High) and Seed shape (Different seed shape was observed as Ellipsoid, Oblong and Reniform).

\section{Results and Discussion}

The essentials of descriptors are that it should be distinctness, uniformity and stability. The descriptors should have distinctiveness i.e., the developed characters should differentiate each hybrids from others, the characters should remain unchanged after repeated propagation or at the end of each cycle (PPV\&FRA, 2006). The descriptors should be stable; it shouldn't vary with in genotypes even at different environmental conditions and it should be uniform, every developed descriptor should be 
regular and unvarying. From the observations made it was found that the Mango genotypes expressed morphological variations from genotype to genotype. These available variations can be developed as descriptors as these morphological variations have the ability to distinguish the Mango genotypes each other.

Descriptors were developed of Mango genotypes after surveying the 24 varieties. Among the genotypes studied, five morphological descriptors were developed and documented, which consist of 27 qualitative characters. Amongst the characters, five descriptors were developed for plant characters, five for inflorescence and fruit characters, eight for leaf characters and four for stone characters have been used.

\section{Plant character}

The mango genotype clone exhibited variations in plant characters (Table 1). All grafted type clones ( 24 clones) varied in their branching pattern. Branching arises from different height from the ground level. In Taimuria clone, primary branches arise from top level of the main stem. Branches arise from basal level in Baramasia and Jawahar. In all the other clones Bombai, Rani Pasand, Husn-e-ara, Dalma, Sundar Prasad, Peri Poona, Alphanso Poona, Mahmood Bhar, Mulgoa, Neeludin, Alfazli, Sabri, Prabhashankar, Menka, Dada Miyan, Kesington, Kurrkkane, Mylepelian, Kesar, Vanraj and Sital Pasand branches arise from intermediate level (Table 1). Tree growth habit also varied in all mango genotypes. The erect type growth observed in Alphanso Poona, Sabri, Menka, Kesington, Kurrkkane and Mylepelian, drooping type growth in Dada Miyan while spreading type were observed in rest genotypes. Similar reports have been suggested by Singh (1968); Dhillon et al., (2004) and Majumder et al., (2011).
Data with respect to crown shape of different clones (Table 1), spherical shape were recorded in Husn-e-ara, Baramasia and Taimuria, broadly pyramidal in Bombai, Rani Pasand, Dalma, Sundar Prasad, Mahmood Bahar, Alfazli, Jawahar, Prabhashankar, Kesar and Sital Pasand, semi- circular in Peri Poona, Neeludin and Vanraj and oblong in Alphanso Poona, Mulgoa, Sabri, Menka, Dada Miyan, Kesington, Kurrkkane and Mylepelian. Different foliage density was observed in all mango genotypes such as dense in Bombai, Baramasia and Prabhashankar, sparse in Taimuria, Peri Poona, Mulgoa, Dada Miyan, Kurrkkane, Mylepelian, Kesar and Vanraj while intermediate in all remaining varieties.

The variation in foliage density of mango varieties might be due to variation in genetic constitution among different cultivars which confirm the earlier finding of Dhillon et al., (2004). Varietal differences in plant growth were noted by Joshi et al., (2013). This variation among different genotypes might be due to the variation in genetic constitution and interaction of various genotypes with agroclimatic conditions. Similar results has been reported by (Barua et al., 2013; Majumder et al., 2011; Mitra et al., 2013) in mango varieties.

\section{Leaf characters}

The leaves colour varied from genotype to genotype and within the varietal variation was observed between young and full grown (mature) leaves. The colours observed in young leaves were observed light green (Bombai, Husn-e-ara, Sunder Prasad, Baramasia, Taimuria, Peri Poona, Alphanso Poona, Neeludin, Jawahar, Prabhashankar, Kesington, Kurrkkane, Mylepelian and Vanraj), reddish brown (Mahmood Bahar, Mulgoa, Menka and Kesington) and light green with brown tinge (Rani Pasand, Dalma, Alfazli, Sabri, Kesar and Sital Pasand). 
Table.1 Plant characters of different mango varieties

\begin{tabular}{|c|c|c|c|c|c|}
\hline Varieties & Tree type & $\begin{array}{c}\text { Branching } \\
\text { pattern }\end{array}$ & $\begin{array}{c}\text { Tree growth } \\
\text { habit }\end{array}$ & Crown shape & $\begin{array}{l}\text { Foliage } \\
\text { density }\end{array}$ \\
\hline Bombai & Grafted & Intermediate & Spreading & Broadly Pyramidal & Dense \\
\hline Rani Pasand & Grafted & Intermediate & Spreading & Broadly Pyramidal & Intermediate \\
\hline Husn-e-ara & Grafted & Intermediate & Spreading & Spherical & Intermediate \\
\hline Dalma & Grafted & Intermediate & Spreading & Broadly Pyramidal & Intermediate \\
\hline Sunder Prasad & Grafted & Intermediate & Spreading & Broadly Pyramidal & Intermediate \\
\hline Baramasia & Grafted & Basal & Spreading & Spherical & Dense \\
\hline Taimuria & Grafted & Top & Spreading & Spherical & Sparse \\
\hline Peri Poona & Grafted & Intermediate & Spreading & Semi-Circular & Sparse \\
\hline $\begin{array}{l}\text { Alphanso } \\
\text { Poona }\end{array}$ & Grafted & Intermediate & Erect & Oblong & Intermediate \\
\hline $\begin{array}{l}\text { Mahmood } \\
\text { Bahar }\end{array}$ & Grafted & Intermediate & Spreading & Broadly Pyramidal & Intermediate \\
\hline Mulgoa & Grafted & Intermediate & Spreading & Oblong & Sparse \\
\hline Neeludin & Grafted & Intermediate & Spreading & Semi-Circular & Intermediate \\
\hline Alfazli & Grafted & Intermediate & Spreading & Broadly Pyramidal & Intermediate \\
\hline Sabri & Grafted & Intermediate & Erect & Oblong & Intermediate \\
\hline Jawahar & Grafted & Basal & Spreading & Broadly Pyramidal & Intermediate \\
\hline Prabhashankar & Grafted & Intermediate & Spreading & Broadly Pyramidal & Dense \\
\hline Menka & Grafted & Intermediate & Erect & Oblong & Intermediate \\
\hline Dada Miyan & Grafted & Intermediate & Drooping & Oblong & Sparse \\
\hline Kesington & Grafted & Intermediate & Erect & Oblong & Intermediate \\
\hline Kurrkkane & Grafted & Intermediate & Erect & Oblong & Sparse \\
\hline Mylepelian & Grafted & Intermediate & Erect & Oblong & Sparse \\
\hline Kesar & Grafted & Intermediate & Spreading & Broadly Pyramidal & Sparse \\
\hline Vanraj & Grafted & Intermediate & Spreading & Semi-Circular & Sparse \\
\hline Sital Pasand & Grafted & Intermediate & Spreading & Broadly Pyramidal & Intermediate \\
\hline
\end{tabular}


Table.2 Leaf characters of different mango varieties

\begin{tabular}{|c|c|c|c|c|c|c|c|c|}
\hline Varieties & Young leaf colour & $\begin{array}{l}\text { Mature leaf } \\
\text { colour }\end{array}$ & $\begin{array}{l}\text { Leaf blade } \\
\text { shape }\end{array}$ & $\begin{array}{l}\text { Leaf apex } \\
\text { shape }\end{array}$ & $\begin{array}{l}\text { Leaf base } \\
\text { shape }\end{array}$ & $\begin{array}{l}\text { Leaf } \\
\text { margin }\end{array}$ & $\begin{array}{l}\text { Angle of sec. } \\
\text { vein } \\
\text { to midrib }\end{array}$ & Leaf texture \\
\hline Bombai & Light green & Green & Oblong & Acuminate & Obtuse & Entire & $45-60^{\circ}$ & Coriaceous \\
\hline Rani Pasand & Light green with brown tinge & Green & Oblong & Acute & Acute & Entire & $45-60^{\circ}$ & Membranous \\
\hline Husn-e-ara & Light green & Green & Elliptic & Acute & Acute & Entire & $45-60^{\circ}$ & Membranous \\
\hline Dalma & Light green with brown tinge & Green & Elliptic & Acuminate & Acute & Entire & $>60^{\circ}$ & Membranous \\
\hline Sunder Prasad & Light green & Green & Ovate & Acuminate & Obtuse & Entire & $>60^{\circ}$ & Chartaceous \\
\hline Baramasia & Light green & Green & Elliptic & $\begin{array}{c}\text { Acute \& } \\
\text { Acuminate }\end{array}$ & Acute & Wavy & $>60^{\circ}$ & Membranous \\
\hline Taimuria & Light green & Green & Elliptic & Acuminate & $\begin{array}{l}\text { Acute \& } \\
\text { Obtuse }\end{array}$ & Entire & $45-60^{\circ}$ & Membranous \\
\hline Peri Poona & Light green & Pale green & Ovate & Acuminate & Obtuse & Entire & $45-60^{\circ}$ & Membranous \\
\hline Alphanso Poona & Light green & Green & Elliptic & Acute & Obtuse & Entire & $>60^{\circ}$ & Chartaceous \\
\hline $\begin{array}{l}\text { Mahmood } \\
\text { Bahar }\end{array}$ & Reddish brown & Green & Elliptic & Acute & Obtuse & Entire & $>60^{\circ}$ & Membranous \\
\hline Mulgoa & Reddish brown & Dark green & Oblong & Acute & Acute & Wavy & $45-60^{\circ}$ & Membranous \\
\hline Neeludin & Light green & Green & $\begin{array}{l}\text { Oblong \& } \\
\text { Lanceolate }\end{array}$ & Acuminate & Acute & Wavy & $45-60^{\circ}$ & Membranous \\
\hline Alfazli & Light green with brown tinge & Green & Elliptic & Acute & Obtuse & Entire & $45-60^{\circ}$ & Chartaceous \\
\hline Sabri & Light green with brown tinge & Green & Elliptic & Acuminate & Acute & Entire & $>60^{\circ}$ & Membranous \\
\hline Jawahar & Light green & Green & Elliptic & Acute & Acute & Entire & $>60^{\circ}$ & Chartaceous \\
\hline Prabhashankar & Light green & Green & Oblong & Acute & Acute & Entire & $45-60^{\circ}$ & Membranous \\
\hline Menka & Reddish brown & Green & Oblong & Acute & Obtuse & Entire & $45-60^{\circ}$ & Membranous \\
\hline Dada Miyan & Light green & Green & Elliptic & Acuminate & Acute & Entire & $>60^{\circ}$ & Membranous \\
\hline Kensington & Reddish brown & Green & Elliptic & Acuminate & Acute & Entire & $>60^{\circ}$ & Membranous \\
\hline Kurrkkane & Light green & Green & Elliptic & Acute & Obtuse & Entire & $45-60^{\circ}$ & Coriaceous \\
\hline Mylepelian & Light green & Green & Elliptic & Acute & Acute & Entire & $45-60^{\circ}$ & Membranous \\
\hline Kesar & Light green with brown tinge & Green & Elliptic & Acuminate & Acute & Entire & $45-60^{\circ}$ & Membranous \\
\hline Vanraj & Light green & Pale green & Elliptic & Acuminate & Acute & Entire & $45-60^{\circ}$ & Chartaceous \\
\hline Sital Pasand & Light green with brown tinge & Pale green & Oblanceolate & Acute & Acute & Entire & $>60^{\circ}$ & Membranous \\
\hline
\end{tabular}


Table.3 Inflorescence characters of different mango varieties

\begin{tabular}{|c|c|c|c|c|c|}
\hline Varieties & $\begin{array}{c}\text { Inflorescence } \\
\text { density }\end{array}$ & Inflorescence colour & $\begin{array}{l}\text { Inflorescence } \\
\text { position }\end{array}$ & $\begin{array}{l}\text { Inflorescence axis } \\
\text { growth habit }\end{array}$ & $\begin{array}{c}\text { Inflorescence } \\
\text { shape }\end{array}$ \\
\hline Bombai & Dense & Pink & Terminal & Horizontal & Pyramidal \\
\hline Rani Pasand & Medium & Green with red patches & Terminal & Semi-erect & Pyramidal \\
\hline Husn-e-ara & Medium & Green with red patches & Terminal & Semi-erect & Conical \\
\hline Dalma & Medium & Green with red patches & Terminal & Semi-erect & Conical \\
\hline Sunder Prasad & Sparse & Light green & Terminal & Horizontal & $\begin{array}{c}\text { Broadly } \\
\text { pyramidal }\end{array}$ \\
\hline Baramasia & Sparse & Green with red patches & Terminal & Semi-erect & Conical \\
\hline Taimuria & Medium & Green with red patches & Terminal & Semi-erect & Pyramidal \\
\hline Peri Poona & Medium & Yellowish green & Terminal & Semi-erect & $\begin{array}{l}\text { Broadly } \\
\text { pyramidal }\end{array}$ \\
\hline Alphanso Poona & Medium & Green with red patches & Terminal & Semi-erect & Conical \\
\hline Mahmood Bahar & Sparse & Light green & Terminal & Semi-erect & Conical \\
\hline Mulgoa & Dense & Light green & Terminal & Semi-erect & Conical \\
\hline Neeludin & Sparse & Green with red patches & Terminal \& Axillary & Semi-erect & Conical \\
\hline Alfarli & Sparse & Light green & Terminal & Horizontal & Pyramidal \\
\hline Sabri & Sparse & Green with red patches & Terminal & Semi-erect & Conical \\
\hline Jawahar & Medium & Yellowish green & Terminal \& Axillary & Semi-erect & Conical \\
\hline Prabhashankar & Medium & Green with red patches & Terminal & Semi-erect & Pyramidal \\
\hline Menka & Medium & Green with red patches & Terminal & Horizontal & Conical \\
\hline Dada Miyan & Medium & Green with red patches & Terminal & Semi-erect & Conical \\
\hline Kensington & Dense & Yellowish green & Terminal & Semi-erect & $\begin{array}{l}\text { Broadly } \\
\text { pyramidal }\end{array}$ \\
\hline Kurrkkane & Medium & Yellowish green & Terminal & Semi-erect & Conical \\
\hline Mylepelian & Dense & Yellowish green & Terminal & Horizontal & Pyramidal \\
\hline Kesar & Sparse & Green with red patches & Terminal & Semi-erect & Conical \\
\hline Vanraj & Medium & Green with red patches & Terminal & Horizontal & Conical \\
\hline Sital Pasand & Sparse & Light green & Terminal \& Axillary & Semi-erect & Conical \\
\hline
\end{tabular}


Table.4 Fruit characters of different mango varieties

\begin{tabular}{|c|c|c|c|c|c|}
\hline Varieties & Fruit colour & $\begin{array}{l}\text { Fruit } \\
\text { shape }\end{array}$ & Fruit size & $\begin{array}{c}\text { Fruit beak } \\
\text { type }\end{array}$ & $\begin{array}{l}\text { Fruit sinus } \\
\text { type }\end{array}$ \\
\hline Bombai & Green & Oblong & Medium & Perceptible & Shallow \\
\hline Rani Pasand & Yellow & Oblong & Small & Perceptible & Absent \\
\hline Husn-e-ara & Yellow with red blush & Oblong & Medium \& long & Perceptible & Shallow \\
\hline Dalma & Green & Oblong & Large & Perceptible & Absent \\
\hline Sunder Prasad & Green with red blush & Roundish & Medium & Perceptible & Shallow \\
\hline Baramasia & Green & Oblong & Medium & Perceptible & Absent \\
\hline Taimuria & Green & Oblong & Long & Perceptible & Absent \\
\hline Peri Poona & Greenish yellow & Oblong & Small & Perceptible & Shallow \\
\hline $\begin{array}{l}\text { Alphanso } \\
\text { Poona }\end{array}$ & Green with red blush & Roundish & Small & Perceptible & Absent \\
\hline $\begin{array}{l}\text { Mahmood } \\
\text { Bahar }\end{array}$ & Green & Oblong & Medium & Perceptible & Absent \\
\hline Mulgoa & Green & Roundish & Small & Perceptible & Shallow \\
\hline Neeludin & Yellow & Oblong & Small & Perceptible & Absent \\
\hline Alfazli & Greenish yellow & Obovoid & Large & Perceptible & Absent \\
\hline Sabri & Yellow & Oblong & Small & Perceptible & Shallow \\
\hline Jawahar & Yellow & Oblong & Small & Perceptible & Absent \\
\hline Prabhashankar & Green & Oblong & Medium & Perceptible & Absent \\
\hline Menka & Yellow & Oblong & Large & Pointed & Absent \\
\hline Dada Miyan & Greenish yellow & Roundish & Small & Pointed & Shallow \\
\hline Kesington & Green with red blush & Roundish & Large & Perceptible & Absent \\
\hline Kurrkkane & Greenish yellow & Roundish & Small & Perceptible & Absent \\
\hline Mylepelian & Yellow & Oblong & Small & Mammiform & Deep \\
\hline Kesar & Greenish yellow & Oblong & Long & Perceptible & Absent \\
\hline Vanraj & Green with red blush & Obovoid & Large & Perceptible & Shallow \\
\hline Sital Pasand & Green & Oblong & Medium & Perceptible & Shallow \\
\hline
\end{tabular}


Table.5 Fruit stone characters of different mango varieties

\begin{tabular}{|c|c|c|c|c|}
\hline Varieties & Veins on stone & $\begin{array}{c}\text { Pattern of stone } \\
\text { venation }\end{array}$ & $\begin{array}{l}\text { Quantity of fibres on } \\
\text { stone }\end{array}$ & Seed shape \\
\hline Bombai & Depressed & Forked & Intermediate & Reniform \\
\hline Rani Pasand & Level with surface & Parallel & Low & Reniform \\
\hline Husn-e-ara & Depressed & Parallel & Low & Oblong \\
\hline Dalma & Depressed & Parallel & Low & Ellipsoid \\
\hline Sunder Prasad & Level with surface & Parallel & Low & Reniform \\
\hline Baramasia & Depressed & Parallel & Low & Reniform \\
\hline Taimuria & Depressed & Forked & Intermediate & Reniform \\
\hline Peri Poona & Elevated & Parallel & Low & Reniform \\
\hline $\begin{array}{l}\text { Alphanso } \\
\text { Poona }\end{array}$ & Elevated & Forked & Intermediate & Reniform \\
\hline $\begin{array}{l}\text { Mahmood } \\
\text { Bahar }\end{array}$ & Elevated & Parallel & Low & Oblong \\
\hline Mulgoa & Level with surface & Parallel & Low & Reniform \\
\hline Neeludin & Elevated & Parallel & Low & Oblong \\
\hline Alfazli & Elevated & Parallel & Low & Reniform \\
\hline Sabri & Level with surface & Parallel & Low & Oblong \\
\hline Jawahar & Level with surface & Parallel & Low & Reniform \\
\hline Prabhashankar & Level with surface & Parallel & Low & Reniform \\
\hline Menka & Elevated & Forked & Low & Reniform \\
\hline Dada Miyan & Level with surface & Forked & Intermediate & Reniform \\
\hline Kesington & Level with surface & Forked & High & Oblong \\
\hline Kurrkkane & Elevated & Forked & Intermediate & Reniform \\
\hline Mylepelian & Elevated & Parallel & Intermediate & Reniform \\
\hline Kesar & Level with surface & Parallel & Low & Reniform \\
\hline Vanraj & Elevated & Forked & Low & Reniform \\
\hline Sital Pasand & Depressed & Parallel & Low & Oblong \\
\hline
\end{tabular}


Similar findings were observed by Fivaz (2008) and Christopher et al., (2017). They also revealed that the immature or young leaves are initially net importers of carbon and only begin to contribute to the carbon economy of the shoot as they expand. Likewise, the mature leaf colour was found dark green in Mulgoa, light green in Peri Poona while green in rests varieties (Table 2). These findings were suggested by Fivaz (2008).

Data reflected that huge variability was noted in leaf blade shape of different genotypes of mango. From Table 2, elliptic shape were observed in Husn-e-ara, Dalma, Baramasia, Taimuria, Alphanso Poona, Mahmood Bahar, Alfazli, Sabri, Jawahar, Dada Miyan, Kesington, Kurrkkane, Mylepelian, Kesar, Mulgoa and Vanraj, ovate shape in Sundar Prasad and Peri Poona, oblong shape in Bombai, Rani Pasand, Mulgoa and Prabhashankar, both Oblong and lanceolate in Neeludin while oblanceolate shape in Sital Pasand. Likewise, acuminate leaf apex shape was found in Bombai, Dalma, Sundar Prasad, Taimuria, Peri Poona, Neeludin, Sabri, Dada Miyan, Kesington, Kesar and Vanraj, both acute and acuminate apex shape in Baramasia whereas acute apex shape in all remaining varieties. Obtuse leaf base shape was observed in Bombai, Sundar Prasad, Peri Poona, Alphanso Poona, Mahmood Bahar, Alfazli, Menka and Kurrkkane, both acute and obtuse shape in Taimuria and all rests observed to have acute leaf base shape. Similar findings have been suggested by Khan (1960); Rhodes et al., (1970); Mussane (2010); Rajwana et al., (2011) and Joshi et al., (2013). From Table 2, entire leaf margin was observed in all 21 genotypes except Baramasia, Mulgoa and Neeludin having wavy leaf margin. Angle of secondary vein to mid rib i.e. $45-60^{\circ}$ of all 14 genotypes but > $60^{\circ}$ angle was observed in Dalma, Sundar Prasad, Baramasia, Alphanso Poona,
Mahmood Bahar, Sabri, Jawahar, Kesington, Dada Miyan and Sital Pasand. Similar works have been reported by numerous scientists (Khan, 1960; Rhodes et al., 1970; Mussane, 2010 and Rajwana et al., 2011). It is found that the leaf texture also changes from genotype to genotype. Bombai was found to have coriaceous texture. Rani Pasand, Husne-ara, Dalma, Baramasia, Taimuria, Peri Poona, Mahmood Bahar, Mulgoa, Neeludin, Sabri, Prabhashankar, Menka, Dada Miyan, Kesington, Mylepelian, Kesar and Sital Pasand were observed with membranous texture and all the other 6 genotypes observed to have chartaceous leaf texture (Table 2).

\section{Inflorescence characters}

Flowering is one of the most important phonological stages of the mango crop production. The type of inflorescence observed in mango varieties was panicle inflorescence. The inflorescence of mango genotypes varied in its density. The flower density observed were dense (Bombai, Mulgoa, Kesington and Mylepelian), medium (Rani Pasand, Husn-e-ara, Dalma, Taimuria, Peri Poona, Alphanso Poona, Jawahar, Prabhashankar, Menka, Dada Miyan, Kurrkkane and Vanraj) and sparse (rest 8 varieties). Flowering intensity is critical factor directly associated with the economic status of the mango growers (Kulkarni, 2004). The inflorescence colour was also observed different in mango genotypes. Pink colour was noticed in Bombai. Light green colour was observed in Sundar Prasad, Mahmood Bahar, Mulgoa, Alfazli and Sital Pasand, yellowish green in Peri Poona, Jawahar, Kesington, Kurrkkane and Mylepelian whereas green with red patches in all other 13 genotypes. These findings have been reported by Hossain and Talukdar (1974). The genotypes were varied in the position of inflorescence. Axillary and terminal positioned inflorescences were observed in 
the genotypes. From Table 3, both terminal and axillary position was noticed in Neeludin, Jawahar and Sital Pasand but terminal position was observed in all other 21 genotypes of mango. This is also in agreement with the findings of Iqbal et al., (1995) and Mazumder et al., (2011). Likewise, the inflorescence axis growth was observed horizontal in Bombai, Sundar Prasad, Alfazli, Mylepelian and Vanraj but semi-erect in rests of the genotypes. Inflorescence shape was noticed pyramidal in Bombai, Rani Pasand, Taimuria, Alfazli, Prabhashankar and Mylepelian, broadly pyramidal in Sundar Prasad and Peri Poona and conical in remaining genotypes (Table 3 ). This difference was also noted by Singh (1968).

\section{Fruit characters}

The mango genotypes having different fruit colour such as green colour in Bombai, Dalma, Baramasia, Taimuria, Mahmood Bahar, Mulgoa, Prabhashankar and Sital Pasand, yellow color in Rani Pasand, Neeludin, Sabri and Jawahar, yellow with red blush in Husn-e-ara, greenish yellow in Peri Poona, Alfazli, Dada Miyan, Kurrkkane and Kesar and green with red blush in remaining genotypes (Table 4). These investigations were carried out by Saha and Hossain (1988) and Haque et al., (1993) under the climatic conditions of southern Bangladesh. This is because fruit colour at maturity is depends upon genotypes reported by Mukherjee (1997), Barholia and Yadav (2014) and Sennhenn et al., (2014).

Shapes of round, oblong and obovoid were observed in different genotypes. Round shape was observed in Sundar Prasad, Mulgoa, Dada Miyan, Kesington and Kurrkkane (5 genotypes) obovoid shape was observed in Alfazli and Vanraj. All the other 17 genotypes were observed with oblong shaped fruits
(Table 4). The reports of Saha et al., (1994) were also in the same tunes under the climatic conditions of Bangladesh. Similar works has also been done by Bhuyan and Islam (1989), Hossain et al., (2002) and Kobra (2007). The size of fruits of mango genotypes also varied from varieties to varieties. Large size fruit was observed in Dalma, Alfazli, Menka, Kesington and Vanraj while small size in Rani Pasand, Peri Poona, Alphanso Poona, Mulgoa, Neeludin, Sabri, Jawahar, Dada Miyan, Kurrkkane and Mylepelian (Table 4). Similar works were also reported by different workers (Singh et al., 1997; Badyal and Bhutani, 1989, Gupta and Brahmachari, 2004 and Chatterjee et al., 2005). According to the findings of Mannan et al., (2003), these variations might be due to the location enjoying different types of environmental conditions, year of production and out crossing among different varieties. Fruit beak was observed pointed in Menka and Dada Miyan, mammiform in Mylepelian and perceptible in rest 21 genotypes. Similar works have also been done in same tunes by Bhuyan and Islam (1989) and Kobra (2007). Likewise, fruit sinus was found absent in Rani Pasand, Dalma, Baramasia, Taimuria, Alphanso Poona, Mahmood Bahar, Neeludin, Alfazli, Jawahar, Prabhashankar, Menka, Kesington, Kurrkkane and Kesar, deep sinus in Mylepelian while shallow sinus in rest genotypes (Table 4). This result is also in consonance with the findings of Hossain and Talukder (1974), Bhuyan and Islam (1989) and Hossain et al., (2002).

\section{Fruit stone characters}

Different genotypes with respect to fruit stones were observed varied from varieties to varieties. Stone veins was found depressed in Bombai, Husn-e-ara, Dalma, Baramasia, Taimuria and Sital Pasand, elevated in Peri Poona, Alphanso Poona, Mahmood Bahar, Neeludin, Alfazli, Menka, Kurrkkane, 
Mylepelian and Vanraj whereas level with surface in all other remained varieties. Likewise, forked stone venation pattern was observed in Bombai, Taimuria, Alphanso Poona, Menka, Dada Miyan, Kesington, Kurrkkane and Vanraj and parallel venation in all other 16 genotypes. Saha et al., (1995) and Majumder et al., (2011) also observed similar variation on the veins on mango stone. Quantity of fibres on stones was high in Kesington, intermediate in Bombai, Taimuria, Alphanso Poona, Dada Miyan, Kurrkkane and Mylepelian while low in rest varieties. These investigations have also been confirmed by Majumder et al., (2011). The shape of seed was also found different such as ellipsoid in Dalma, oblong in Husn-e-ara, Mahmood Bahar, Neeludin, Sabri, Kesington and Sital Pasand and reniform in all 17 genotypes. Similar works were observed by Jilani et al., (2010) (Table 5).

A research work was taken up with the objective to develop and document descriptors for Mango and its genotype derivatives, which enables the identification of varieties for varietal registration and will also help tree improvement programmes. Characterization of clones through morphological traits is the simplest, quickest and easily adoptable method for identification of clones at the field level which is mandatory for tree improvement programmes. Mango genotypes were identified based on morphological characters by developing descriptors and documenting the developed ones. Descriptors were developed based on the guidelines that are envisaged in PPA\&FA act 2001. Accordingly in present study, major focus was given to develop descriptors for some characters of plant, leaf, inflorescence, fruit and stone. Descriptors were developed for Mango genotypes after surveying the 24 varieties developed in Experimental plot of Horticulture Garden, Bihar Agricultural University, Sabour, Bhagalpur. Among the varieties studied, five morphological descriptors were developed and documented, which consist of 27 qualitative characters. Among the characters, five descriptors were developed for plant characters which include plant type; branching pattern; plant growth habit; crown shape and foliage density; eight descriptors for leaf characters which include young leaf colour; mature leaf colour; leaf blade shape; leaf apex shape; leaf base shape; leaf margin; angle of secondary vein to mid rib and leaf texture; five characters for inflorescence which admits flower density; inflorescence colour; inflorescence position; inflorescence axis growth habit and inflorescence shape, five characters for fruit which include fruit colour, shape; size; beak and sinus and four characters for seed which admits veins on stone; stone venation pattern; fibre quantity on stone and seed shape.

\section{References}

Anumalla, M., Roychowdhury, R., Geda, C. K., Mazid, M. and Rathoure, A. K. 2015. Utilization of plant genetic resources and diversity analysis tools for sustainable crop improvement with special emphasis on Rice. International Journal of Advanced Research, 3(3): 1155-1175.

Badyal, J. and Bhutani, V. P. 1989. Physicochemical characteristics of some mango cultivars under Sub-mountaneous regions of Himachal Pradesh. Haryana J. Hort. Sci., 18 (1 \& 2): 51-55.

Barholia, A.K. and Yadav, S. 2014. Divergence for fruit characters in mango (Mangifera indica L.). Afr. $J$. Agric. Sci. Technol., 2:65-67.

Barua, H., Alam, M., Patwary, M. and Rahman, M. H. 2013. Performance of Bari mango (Mangifera indica L.) varieties in Chittagong region. Bangladesh J. Agril. Res., 38(2): 203209. 
Bhuyan, M. A. J. and Islam, M. S. 1989. Physio-morphological characters of some popular mango cultivars. Bangladesh J. Agric., 14(3): 181-187.

Biodiversity International. 2007. Guidelines for the development of crop descriptor lists. Biodiversity Technical Bulletin Series. Biodiversity International, Rome, Italy. ISBN: 978-92-9043-792-1. Chatterjee, D., Maurya, K. R. and Mandal, M. P. 2005. Physico-chemical characteristics of mango (Mangifera indica L.). The Orissa J. Hort., 35 (2): 57-59.

Christopher, M., Menzela, M. D. and Lagadec, L. 2017. Can the productivity of mango orchards be increased by using high-density plantings? Scientia Horticulturae, 219: 222-263.

De Vicente, M. C., Guzma'n, F. A., Engels, J. and Rao, V. R. 2006. Genetic characterization and its use in decision making for the conservation of crop germplasm. In: Ruane J, Sonnino A(eds) The role of biotechnology in exploring and protecting agricultural genetic resources. Food and Agriculture Organization, Rome, pp 121-128.

Degani, C., Cohen, M., El-Batsri, R. and Gazit, S. 1992. PGI isozyme diversity and its genetic control in mango. Horticultural Science, 27:252-254.

Dhillon, W. S., Sharma, R. C. and Kahlon, G. S. 2004. Evaluation of some mango varieties under Punjab conditions. The Haryana J. Hort., 33 (3 \& 4):157-159.

Fivaz, J. 2008. Botanical aspects. In: de Villiers, E.A., Joubert, P.H. (Eds.), The Cultivation of Mango. ARC Institute for Tropical and Subtropical Crops, Florida, USA, pp. 9-20.

Gupta, R. K. and Brahmachari,V. S. 2004. Effect of foliar application of urea, potassium nitrate and N.A.A on fruit retention, yield and quality of mango $c v$. Bombai. Orissa, J. Hort., 32(2).
Hammer, K., Arrowsmith, N. and Gladis, T. 2003. Agrobiodiversity with emphasis on plant genetic resources. Naturwissenschaften, 90: 241-250.

Haque, A. M. M., Ali, M. R., Uddin, M. R. and Hossain, A. K. M. A.1993. Evaluation of elite mango cultivars at southern region of Bangladesh. Bangladesh J. Plant Breed. Gent., 6(2): 21-28.

Hossain, M. A. and Talukdar, M. R. 1974. Characteristics of Bangladeshi mangos grown at Rajshahi. An. M. Sc. (Ag) Thesis, Dept, of Horticulture, Bangladesh Agricultural University, Mymensingh. 103p.

Hossain, M. A., Rafiuddin, M., Hasan, M. K., Malaker, P. K. and Hossain, M. A. 2002. Morphological and physicochemical studies of seventeen mango varieties. Bangladesh J. Agril. Res., 27(2): 273-281.

Iqbal, S. M., Uddin, M. S. and Sharker, M. A. 1995. Performance of exotic mango germplasm under Bangladesh conditions. In: Annual Report on Mango Improvement, Regional Horticultural Research Station, BARI, Nawabgonj. pp. 1-9.

Jilani, M. S., Bibi, F., Waseem, K. and Khan, M. A. 2010. Evaluation of physicochemical characteristics of mango (Mangifera indica L.) cultivars grown in D. L. Khan. J. Agric. Res, 48(2): 201-207.

Joshi, R., Kundu, M. and Singh, C. P. 2013. Morphological Characters: Efficient Tool for Identification on Different Mango Cultivars. Environment \& Ecology, 31(1A):385-388.

Khan, A. A. 1960. Relation of growth to fruit bearing in mangoes. Punjab Fruit J., 23:117-140.

Kostermans, A. J. G. H. and Bompard J. M. 1993. The mangoes. Botany, nomenclature, horticulture, and 
utilization. Academic Press, London, UK.

Kulkarni, V. J. 2004. The tri-factor hypothesis for flowering in mango. Acta Hortic., 645:61-70.

Majumder, D. A. N., Hassan, L., Rahim, M. A. and Kabir, M. A. 2011. Studies on physio-morphology, floral biology and fruit characteristics of mango. $J$. Bangladesh Agril. Univ., 9(2):187-199.

Mannan, M. A., Khan, S. A. K. U., Islam, M. R., Islam, M. S. and Siddiqua, A. 2003. A Study on the Physico-chemical Characteristics of Some Mango Varieties in Khulna Region. Pakistan Journal of Biological Sciences, 6(24):2034-2039.

Mitra, S. K., Ghosh, B. and Pathak, P. K. 2013. ISHS Acta Horticulturae 992: IX International Mango Symposium. Mango cultivars and hybrids grown in West Bengal, India. Acta Hortic. 992, $43 p$.

Mukherjee, S. K. 1997. Introduction: botany and importance. In: The mango: Botany, Production and Uses. 1st edn. (Ed. R. E. Litz), CAB International, Wallingford, UK. pp. 1-19.

Mukherjee, S. K. 1953. Origin, distribution, and phylogenetic affinity of the species of Mangifera L. Journal of the Linnean Society, Botany, 55:65-83.

Mussane, C. R. B. 2010. Morphological and Genetic Characterization of Mango (Mangifera indica L.) varieties in Mozambique. M.Sc. Thesis. University of the Free State, Bloemfontein, South Africa.

N H B. 2015. National Horticulture Board data base. (http://nhb.gov.in/)

PPV\&FRA. 2006. Proceedings of the First Meeting of the Protection of Plant Varieties and Farmers' Rights (PPV\&FR) Authority held on 18th May 2006 in the Committee Room of PPV\&FR Authority, under the
Chairmanship of Chairperson of PPV\&FR Authority.

Rajwana, I. A., Khan, I. A., Malik, A. U., Saleem, B. A., Khan, A. S., Ziaf, K., Anwar, R. and Amin, M. 2011. Morphological and bio-chemical markers for varietal characterization and quality assessment of potential indigenous mango (Mangifera indica L.) germplasm. Int. J. Agric. Biol., 13:151-158.

Rhodes, M. A., Campbell, G., Malo, S. E. and Camer, S. G. 1970. A numerical taxonomic studies of the mango (Mangifera indica L.). J. Am. Soc. Hortic. Sci., 95:252-256.

Rubenstein, D. K., Smale, M. and Widrlechner, M. P. 2006. Demand for genetic resources and the U.S. National Plant Germplasm System. Crop Sci., 46:1021-1031.

Saha, N. N., Bhuyan, M. A. J. and Islam, M. S. 1995. Variability in early and mid seasons mango varieties grown in Bangladesh. Annals Bangladesh Agric., 5(2): 135-138.

Saha, N. N., Bhuyan, M. A. J., Islam, M. S. and Hossain, A. K. M. A. 1994. Fruit characteristics of some late mango (Mangifera indica) varieties of Bangladesh. Bangladesh Agric., 4(1): 25-30.

Saha, S. K. and Hossain, A. K. M. A. 1988. Studies of some grafted mango cultivars. Bangladesh J. Agril. Res., 13(2): 47-52.

Sankar, J., Rosaiah, G., Kurapati, R. K. and Pinnamaneni, R. 2011 Molecular Identification of mango, Mangifera indica $\mathrm{L}$. var. Totupura. Bioinformation, 5:405-409.

Sennhenn, A., Prinz, K., Gebauer, J., Whitbread, A., Jamnadass, R. and Kehlenbeck, K. 2014. Identification of mango (Mangifera indica L.) landraces from Eastern and Central Kenya using a 
morphological and molecular approach. Genet. Res.Crop. Evol., 61:7-22.

Singh, L. B. 1968. The Mango: Botany, Cultivation and Utilization. Leonard Hill, London. 438p.

Singh, L. B. 1969. Mango (Mangifera indica L.) In: Outlines of Perennial Crop Breeding in the Tropics, (F.P. Ferwerda and F. Wit Eds.) Landbouwhogeschool, Wageningen, Netherlands, pp. 309-327.
Singh, S., Singh, R. and Kumar, J. 1997. Evaluation of shelf-life of plum as affected by some chemicals. Haryana J. Hort. Sci., 26(3-4): 194-199.

UPOV. 1989. Guidelines for the conduct of tests for distinctness, homogeneity and stability. Banana (Musa acuminata Colla). TG/123/3. International Union for the Protection of New Varieties and Plants (UPOV), Geneva. 26 p.

\section{How to cite this article:}

Kanchan Bhamini, Anjani Kumar, U.S. Jaiswal, Md. Feza Ahmad and Ruby Rani. 2018. Morphological Characterization of Mango (Mangifera indica L.) Germplasm Using DUS Testing. Int.J.Curr.Microbiol.App.Sci. 7(05): 2944-2959.

doi: https://doi.org/10.20546/ijcmas.2018.705.343 